OPEN ACCESS

Edited by:

Marian Elizabeth Ludgate, Cardiff University, United Kingdom

Reviewed by: Yun-Bo Shi, National Institutes of Health (NIH),

United States

Dorina Ylli,

MedStar Health Research Institute (MHRI), United States

*Correspondence:

Huijjuan Wang

whj@nwu.edu.cn

Guifeng Ding

dingguifeng123@126.com

${ }^{t}$ These authors have contributed equally to this work

Specialty section:

This article was submitted to

Thyroid Endocrinology,

a section of the journal

Frontiers in Endocrinology

Received: 13 September 2021 Accepted: 24 November 2021 Published: 21 December 2021

Citation:

Wang $H$, Wang W, Chen $X$ Shi H, Shi $Y$ and Ding G (2021) Screening and Functional Analysis

of TPO Gene Mutations in a

Cohort of Chinese Patients With

Congenital Hypothyroidism.

Front. Endocrinol. 12:774941. doi: 10.3389/fendo.2021.774941

\section{Screening and Functional Analysis of TPO Gene Mutations in a Cohort of Chinese Patients With Congenital Hypothyroidism}

\author{
Huijjuan Wang ${ }^{1 \star t}$, Wenxia Wang ${ }^{1 \dagger}$, Xi Chen $^{2}$, Hailong Shi ${ }^{3}$, Yinmin Shi ${ }^{1}$ \\ and Guifeng Ding ${ }^{4 *}$ \\ 1 The National Engineering Research Center for Miniaturized Detection Systems, Northwest University, Xi'an, China, \\ 2 Pediatrics, Urumqi Maternal and Child Health Care Hospital, Urumqi, China, ${ }^{3}$ College of Basic Medicine, Shaanxi University \\ of Chinese Medicine, Xianyang, China, ${ }^{4}$ Obstetrics and Gynecology Department, Urumqi Maternal and Child Health Care \\ Hospital, Urumqi, China
}

Backgrounds: As a crucial enzyme in thyroid hormone synthesis, the genetic defective thyroid peroxidase (TPO) was one of the main genetic factors leading to congenital hypothyroidism $(\mathrm{CH})$.

Methods: Mutations in the TPO gene were screened and identified in 219 patients with $\mathrm{CH}$ from northwest China by using high-throughput sequencing and bioinformatics analysis. The biological function of detected variants was studied by in vitro experiments and homology modeling.

Results: Nineteen rare variants, including seven novel ones, were detected in 17 of 219 patients (7.8\%). Most cases were detected with one single heterozygous variant, and only two patients were detected with multiple variants, i.e., compounds for (1) IVS7-1G>A, p.Ala443Val, and p.Arg769Trp and (2) p.Asn592Ser and p.Asn798Lys. The biological function of the four missense mutations (i.e., p.Ala443Val, p.Arg769Trp, p.Asn592Ser, and p.Asn798Lys) they carried were further studied. Experimental data showed that these four mutations did not affect the protein expression level of the TPO gene but remarkably reduced the peroxidase activity toward guaiacol oxidation, retaining $8-32 \%$ of activity of the wild-type protein. The comparison of the predicted 3-D structures of wild-type and mutant TPO proteins showed that these four amino acid substitutions changed the noncovalent interactions of studied residues that might alter the structure and function of the TPO protein.

Conclusion: This study was the first to analyze the TPO mutation spectrum of patients with $\mathrm{CH}$ in northwest China. Our data indicated that the TPO mutation was not a common reason to cause $\mathrm{CH}$ in China. The functional data may help to clarify the structure-function relationship of the TPO protein and provide further evidence for the elucidation of the genetic etiology of $\mathrm{CH}$.

Keywords: congenital hypothyroidism (CH), thyroid peroxidase (TPO), gene mutation, genotype, phenotype 


\section{INTRODUCTION}

Congenital hypothyroidism $(\mathrm{CH})$ is one of the most frequent neonatal endocrine and metabolic diseases and affects approximately 1:2,000-1:4,000 infants worldwide (1). As a preventable complex disease, highly variable clinical manifestations and symptoms are observed among different individuals $(1,2)$. Although the precise pathogenic mechanism of $\mathrm{CH}$ is not elucidated, a genetic origin in the pathogenesis has been widely acknowledged, and a proportion of patients have hereditary diseases caused by genetic abnormalities (3-5). To date, a number of genes associated with $\mathrm{CH}$ have been identified $(6,7)$. Genetic defective thyroid peroxidase (TPO) is considered as one of the most common causes of thyroid dyshormonogenesis $(1,4)$.

TPO is a thyroid-specific heme peroxidase localized in the apical membrane of thyrocytes that catalyzes the oxidation and organization of iodide in the tyrosine residues of thyroglobulin and forms active thyroid hormones by the coupling of iodotyrosine residues $(8,9)$. Considering the key role of TPO in thyroid hormone biosynthesis, mutations in the TPO gene can impair thyroid hormone production due to total (TIOD) or partial (PIOD) iodide organification defects $(9,10)$. Cases of $\mathrm{CH}$ due to TPO genetic defects are usually inherited in an autosomal recessive manner and are often manifested as permanent $\mathrm{CH}$ and goiter (9-11). Severe defective TPO bioallelic mutations were considered to lead to TIOD, and PIOD is generally related to the heterozygous TPO mutation $(9,12)$. However, single TPO mutations were detected in some patients with TIOD, and multiple TPO mutations were detected in some patients with PIOD, indicating a lack of direct correlation between TPO genotypes and phenotypes $(9,12-14)$. In addition, the frequency and distribution of TPO mutations in patients with $\mathrm{CH}$ seem to be specific to ethnicity. In patients with thyroid dyshormonogenesis, the detection rate of TPO mutations is 20$46 \%$ in Caucasians (15-17) but only less than 10\% in East Asians (18-23). To date, more than 170 mutations in the TPO gene have been recorded in the Human Gene Mutation Database (HGMD professional, 2021.2). However, the biological function of most of the identified TPO mutations remains unknown. Therefore, comprehensive studies covering different populations and indepth functional studies are helpful to have a thorough understanding of the role of TPO mutation in the pathogenesis of $\mathrm{CH}$.

According to the newborn screening data, the incidence rate of $\mathrm{CH}$ in China is higher than the global average level $(24,25)$ and is even higher in northwest China. A rate of 1 in 1,468 newborns was reported in Xinjiang (26), which accounts for onesixth of China's territory. Currently, the TPO mutation spectrum in patients with $\mathrm{CH}$ of these regions is not studied yet. Therefore, in this study, TPO mutations in 219 patients with $\mathrm{CH}$ from northwest China are comprehensively screened using highthroughput sequencing to obtain the TPO mutation spectrum of patients with $\mathrm{CH}$ in northwest China and explore the genotype-phenotype relationship. Moreover, the function of some important mutations is investigated through a series of in vitro experiments and molecular modeling. The findings obtained in this study will facilitate the genetic counseling and prognosis of patients with $\mathrm{CH}$ and will be helpful for clarifying the molecular mechanism underlying $\mathrm{CH}$ pathogenesis caused by TPO mutation(s).

\section{MATERIALS AND METHODS}

\section{Study Samples}

A total of 219 patients with $\mathrm{CH}$ were recruited in Urumqi Maternal and Child Health Care Hospital, Xinjiang, China, from October 2017 to June 2019. The inclusion criteria were as follows: positive neonatal screening with a diagnosis of $\mathrm{CH}$ confirmed by serum thyroid function tests at $2-4$ weeks of age (27). In brief, all patients undergoing neonatal screening with TSH levels of $>10 \mathrm{uIU} / \mathrm{ml}$, elevated serum TSH $(>7.5 \mathrm{uIU} / \mathrm{ml})$, and decreased FT4 levels $(0.5-7.1 \mathrm{pmol} / \mathrm{L})$ at diagnosis were finally included. Thyroid ultrasonography and $99 \mathrm{mTc}$ scintigraphy were performed during the neonatal period prior to treatment. Patients with thyroids width $\geq+2 S D$ (standard error) in size were defined as goiters, with thyroids $\leq-2 \mathrm{SD}$ were considered as hypoplasia. The parents of all participants gave written informed consent in accordance with the Declaration of Helsinki. The study was approved by the Medical Ethics Committees of Urumqi Maternal and Child Health Care Hospital.

\section{DNA Extraction and Sequencing}

Genomic DNA was extracted from the peripheral blood samples collected from recruited patients. Recruited patients were genetically screened with a customized Ampliseq panel that included $29 \mathrm{CH}$-associated genes as described previously (27). The coding exons and the 20 flanking base pairs of splice junctions surrounding the exons of the targeted genes were sequenced using the Ion Torrent PGM instrument (Thermo Fisher Scientific, MA, USA). For the TPO gene, 21 amplified amplicons were obtained at each sequencing run. The amplicon length ranged from $166 \mathrm{bp}$ to $374 \mathrm{bp}$.

\section{Variant Detection and Interpretation}

Raw data were processed using the Torrent Suite software (version 5.0.4; Life Technologies), and the Ion Reporter (https://ionreporter.lifetechnologies.com/ir/secure/home.html) and ANNOVAR package (http://wannovar.wglab.org/) were used for variant annotation. Variants were filtered in accordance with the criteria described in our published study (27). In brief, non-synonymous variants with minor allele frequencies (MAFs) of $\leq 0.01$ or no MAF values in the public databases, such as dbSNP database (http://www.ncbi.nlm.nih. gov/projects/SNP/), 1000 Genomes Project (http://ftp.ncbi.nih. gov/), Exome Sequencing Project (http://evs.gs.washington.edu/ EVS/), and the Genome Aggregation Database (http://gnomad. broadinstitute.org/), were selected. All selected variants were validated through the Sanger sequencing with the ABI3500 xL Dx (Applied Biosystems, Foster City, CA, USA).

The effects of detected variants were assessed using in silico programs. For missense or indel variants, four in silico tools, 
including Polymorphism Phenotyping v2 (http://genetics.bwh. harvard.edu/pph2/index.shtml), Mutation Taster (http://www. mutationtaster.org/), SIFT (http://provean.jcvi.org/genome_ submit_2.php), and Mendelian Clinically Applicable Pathogenicity (http://bejerano.stanford.edu/MCAP/), were used. The pathogenicity of each variant was assessed in accordance with the standards described by the American College of Medical Genetics (ACMG) (28).

The protein sequences of TPO from various species and other members of the peroxidase superfamily were downloaded from NCBI (https://www.ncbi.nlm.nih.gov/protein/). Alignments were performed using the DNAMAN version 9 (Lynnon Biosoft, Quebec, Canada). The protein domain and structure of TPO were obtained from the UniProt Knowledgebase (https:// www.uniprot.org/).

\section{Homology Modeling of Human TPO Protein}

A three-dimensional homology model of human TPO was constructed using the Modeller 9.13 software (https://salilab.org/ modeller/). The crystal structure of Human Myeloperoxidase (PDB id: 6BMT) was used as template for TPO homology modeling because of the highest amino acid homology (42\% sequence similarity). The same software was used to generate a homology model of the human TPO containing the detected mutation for structural comparison. Both models were then subjected to molecular dynamics simulation by using the Gromacs 2019 software (https://manual.gromacs.org/) with CHARMM27 allatom force field and explicit water environment neutralized by counter ions at $310 \mathrm{~K}$. Structures with the lowest potential energy were extracted and verified using Ramachandran plots, which were generated using the Procheck software (http://nihserver.mbi.ucla. edu/SAVES), to check the stereochemical quality of the protein. The Verify3D software was used to determine the compatibility of the $3 \mathrm{D}$ atomic model with its own $1 \mathrm{D}$ amino acid sequence, and the Errat software (http://nihserver.mbi.ucla.edu/SAVES) was used to analyze the statistics of non-bonded interactions among different atom types. The BIOVIA Discovery Studio Visualizer (www.3ds. com, Dassault Systèmes; San Diego, CA, USA) was used for viewing, sharing, and analyzing protein data.

\section{In Vitro Functional Analysis of TPO Mutants}

Th pEGFP-C1-based vectors containing TPO genes with different genotypes, including wild-type (wt) and five mutant TPO (i.e., p.Glu337Lys, p.Ala443Val, p.Asn592Ser, p.Arg769Trp, and p.Asn798Lys), were constructed. HEK 293T cells were maintained in Dulbecco's Modified Eagle Medium (Gibco, Life Technologies) supplemented with $50 \mathrm{U} / \mathrm{ml}$ penicillinstreptomycin, $2 \mathrm{mM}$ L-glutamine, and 10\% fetal bovine serum (Gibco, LifeTechnologies). Cells were seeded in a 24 -well plate $\left(1 \times 10^{5}\right.$ cells per well) and transfected with plasmids by using the Lipofectamine 2000 (Invitrogen). Cells were harvested after $24 \mathrm{~h}$ transfection, washed twice with phosphate buffered saline (PBS), and lysed using a sonicator in PBS $(\mathrm{pH}=7.4)$ in ice. Sonicated extracts were subjected to centrifugation for $5 \mathrm{~min}$ at $1,000 \mathrm{~g}$ $\left(4^{\circ} \mathrm{C}\right)$. The supernatant was transferred into a new tube and quantified through the Bradford assay (HyClone-Pierce, USA) by using bovine serum albumin as a standard in accordance with the manufacturer's protocol.

For immunoblot analysis, $60 \mu \mathrm{g}$ supernatant prepared from the cells transfected for $24 \mathrm{~h}$ were separated by $10 \%$ SDS-PAGE. Western blotting was performed with an anti-His tag rabbit monoclonal antibody (Cell Signaling Technology, MA, USA) used at 1:1,000 dilution. Horseradish peroxidase-labeled goat antirabbit antibody $\operatorname{IgG}$ (1:5,000 dilution; ABclonal, China) was used as a second antibody. Immunoreactive proteins were visualized using the SuperSignal West Femto Trial Kit (Thermo Fisher Scientific Inc., Rockford, IL, USA), and band densities were determined with the BIO-1D software (SIM International Group Co., Newark, NJ, USA). The relative protein level of each detected sample was corrected to the $\beta$ actin expression and expressed in relation to wt TPO protein (i.e., relative protein level of wt TPO was 100\%). All assays were performed in triplicate with independent preparations.

The guaiacol oxidation assay was performed to measure the enzymatic activity of wt and mutant TPO proteins (29). The reaction mixture contained $10 \mu \mathrm{l}$ supernatant, $5 \mathrm{mmol} / \mathrm{L}$ guaiacol, and $892 \mathrm{mM} \mathrm{H}_{2} \mathrm{O}_{2}$ in PBS. The reaction was initiated with the addition of $\mathrm{H}_{2} \mathrm{O}_{2}$, and absorbance was monitored spectrophotometrically at $470 \mathrm{~nm}$ every $60 \mathrm{~s}$ for more than 30 min. The reaction velocity was estimated from the slope of the initial linear part $(d \mathrm{~A} / d \mathrm{t})$ of the curves obtained, and the specific enzyme activity of each tested sample was expressed as $d \mathrm{~A} \cdot \mathrm{min}^{-1} \cdot \mathrm{mg}^{-1}$. Data were representative of at least three independent experiments (each performed in triplicate) with similar results.

\section{Statistical Analysis}

Statistical analysis was performed by using the GraphPad Prism software (version 5.01; GraphPad Software, Inc., San Diego, CA, USA). The one-way analysis of variance with Dunnett's test was used to evaluate the differences in the enzymatic activity between wt and mutant TPO enzymes. $\mathrm{P}<0.05$ was considered statistically significant.

\section{RESULTS}

\section{TPO Mutation Spectrum Analysis}

Through the genetic screening of 219 patients with $\mathrm{CH}, 19$ rare variants (with $\mathrm{MAF}<1 \%$ in public population databases) in the TPO gene were found in 17 subjects with a mutation detection rate of $7.76 \%(17 / 219)$. These variants included 17 missense mutations, 1 non-sense mutation, and 1 splicing variant. Of these variants, 12 were reported in the published literature and databases. Six of these variants were identified in the reported cases of published studies and considered as (likely) pathogenic mutations, and the six other variants were reported in the public population databases but not reported in patients. Seven variants, including 1 splicing variant (i.e., IVS7-1G>A) and 6 missense mutations (i.e., p.Arg279Trp, p.Ser309Pro, 
p.Ala443Val, p.Ser571Arg, p.Asn798Lys, and p.Ser853Leu;

Tables 1 and 2), were first found in this study.

Most of the detected variants were only found in one patient, whereas p.Pro883Ser and p.Arg846Trp were found in two cases. c.2268dupT, which was reported as a hotspot mutation in Taiwanese and Malaysian patients with $\mathrm{CH}(10,30)$, was only found in one patient. Majority of patients carried one heterozygous variant, and only two cases (E160098 and E170009) carried multiple heterozygous variants (Table 1), i.e., compounds for (1) IVS7-1G>A, p.Ala443Val, and p.Arg769Trp and (2) p.Asn592Ser and p.Asn798Lys. The family pedigree analysis showed that of the variants harbored by E160098, IVS7$1 G>A$ was inherited from her father, and p.Ala443Val and p.Arg769Trp were inherited from her mother. Of the variants carried by E170009, p.Asn592Ser was inherited from her father, and p.Asn798Lys was inherited from her mother (Figure 1).

Of the 17 cases with TPO variants, 9 presented normal thyroid shape and size, 6 were diagnosed with goiter, 1 showed thyroid dysplasia, and 1 was suspected with athyreosis. On the basis of serum FT4 levels at diagnosis, $\mathrm{CH}$ could be categorized as severe (FT4 $<5 \mathrm{pmol} / \mathrm{L})$, moderate $(5 \mathrm{pmol} / \mathrm{L} \leq \mathrm{FT} 4<10 \mathrm{pmol} / \mathrm{L})$, or mild (FT4 $\geq 10 \mathrm{pmol} / \mathrm{L}$ ) (31). The two cases with multiple TPO variants were severe $\mathrm{CH}$ but with normal thyroid. Of the cases carrying one variant, 9 had moderate or mild $\mathrm{CH}$, and 6 had severe $\mathrm{CH}$. Two cases had the same mutation p.Pro883Ser, but their phenotype was different. The first case had moderate $\mathrm{CH}$ with goiter, and the other case had severe $\mathrm{CH}$ with athyreosis.

\section{Pathogenicity Evaluation of Detected Variants}

On the basis of available evidence, the pathogenicity of detected variants was classified in accordance with the ACMG standards and guidelines. Of 19 detected variants, 10 variants were classified as pathogenic (P) or likely pathogenic (LP), including IVS7-1G>A, p.Glu757*, p.Pro883Ser, p.Ser853Leu, p.Asn798Lys, p.Arg769Trp, p.Asn592Ser, p.Ala443Val, p.Arg361Leu, and p.Glu337Lys. Other nine variants were classified as variant of uncertain significance (VUS). Among the seven novel variants, four (IVS7-1G>A, p.Ala443Val, p.Asn798Lys, and p.Ser853Leu) were evaluated as P or LP, and the other was classified as VUS (Table 2 and Supplementary Table 1).

\section{In Silico Analysis of Effects of the Detected Variants}

Of the detected variants, p.Pro135His was located at the $\mathrm{N}$ terminal of TPO protein, and p.Gly889Arg and Pro883Ser were located at the C-terminal tail (intracellular domain). p.Asn798Lys was located at the epidermal growth factor (EGF)-like domain; p.Arg769Trp and p.Glu757* were located at the complement control protein (CCP)-like domain; p.Asn674Ser, p.Gly673Lys, p.Gly650Glu, p.Asn592Ser, p.Ser571Arg, p.Ala443Val, p.Arg361Leu, p.Glu337Lys, p.Ser309Pro, p.Arg279Trp were located at the myeloperoxidase (MPO)-like domain (catalytic domain); and IVS7-1G $>A$ was located in the intron near the catalytic active center (Figure 2).

The possible functional effects of detected missense mutations were assessed by in silico programs, including SIFT, PolyPhen-2, MutationTaster, and M-CAP. As shown in Supplementary Table 2, p.Gly673Lys, p.Gly650Glu, p.Asn592Ser, and p.Ala443Val were predicted by all software programs to have a deleterious effect on the TPO protein, whereas p.Pro883Ser was harmless to the protein function. The prediction results of other variants were inconsistent among different software programs.

The 3D homology model of the wt TPO and four mutant (i.e., p.Asn798Lys, p.Arg769Trp, p.Asn592Ser, and p.Ala443Val) proteins were constructed using in silico tools (Figure 3 and

TABLE 1 | Clinical information of $\mathrm{CH}$ patients with TPO variants.

\begin{tabular}{|c|c|c|c|c|c|c|c|c|c|c|c|}
\hline \multirow[t]{2}{*}{$\begin{array}{l}\text { Patient } \\
\text { ID }\end{array}$} & \multirow[t]{2}{*}{$\begin{array}{l}\text { Date of } \\
\text { birth }\end{array}$} & \multirow[t]{2}{*}{$\begin{array}{c}\text { Birth } \\
\text { weight }(g)\end{array}$} & \multirow[t]{2}{*}{$\begin{array}{c}\text { Gestational } \\
\text { age (week+day) }\end{array}$} & \multirow[t]{2}{*}{ Gender } & \multirow[t]{2}{*}{ Thyroid } & \multicolumn{2}{|c|}{$\begin{array}{l}\text { Neonatal } \\
\text { screening }\end{array}$} & \multicolumn{2}{|c|}{ Diagnostic } & \multirow[t]{2}{*}{ Severity } & \multirow[t]{2}{*}{ TPO mutation } \\
\hline & & & & & & $\begin{array}{c}\text { TSH } \\
\text { (ulU/ml) }\end{array}$ & Age & $\begin{array}{c}\text { TSH } \\
\text { (ulU/ml) }\end{array}$ & $\begin{array}{c}\text { FT4 } \\
\text { (pmol/L) }\end{array}$ & & \\
\hline E150012 & 2012/1/2 & 2,900 & 37 & Female & Hypoplasia & 35.4 & $69 d$ & $>100$ & 1.20 & Severe & p.Arg361Leu \\
\hline E150018 & $2014 / 10 / 29$ & 3,500 & 41 & Male & Normal & $>100$ & $20 d$ & $>100$ & 0.70 & Severe & p.Glu757* \\
\hline E150027 & 2014/4/15 & 3,500 & $40+6$ & Male & Goiter & 19 & $61 d$ & 58.21 & 4.50 & Moderate & p.Arg846Trp \\
\hline E150041 & $2009 / 11 / 14$ & 3,000 & 37 & Male & Goiter & 18 & $58 d$ & 20 & 6.40 & Mild & p.Ser571Arg \\
\hline E150048 & $2014 / 10 / 24$ & 3,750 & $41+2$ & Male & Goiter & 35.1 & $78 d$ & 35 & 4.90 & Moderate & p.Pro883Ser \\
\hline E160002 & 2014/11/8 & NA & NA & Female & Athyreosis & 46.1 & $64 d$ & $>100$ & 1.90 & Severe & p.Pro883Ser \\
\hline E160006 & $2015 / 5 / 16$ & 1,900 & 38 & Female & Normal & 15 & $33 d$ & 15 & 7.10 & Mild & p.Ser309Pro \\
\hline E160053 & 2014/3/2 & 3,950 & $41+4$ & Male & Normal & 223 & $19 d$ & $>100$ & 3.73 & Severe & p.Pro135His \\
\hline E160078 & $2015 / 7 / 8$ & 3,100 & NA & Female & Goiter & 44 & $24 d$ & 15.33 & 2.34 & Severe & p.Asn674Ser \\
\hline E160089 & 2016/6/20 & 3,300 & 42 & Male & Normal & 11.9 & $25 d$ & 13.4 & 8.80 & Moderate & p.Gly889Arg \\
\hline E160095 & $2015 / 7 / 5$ & 3,800 & 40 & Male & Goiter & $>100$ & $74 d$ & $>100$ & 0.60 & Moderate & p.Arg846Trp \\
\hline E160098 & 2011/8/16 & 3,780 & 40 & Female & Normal & 646 & $28 d$ & $>100$ & 1.29 & Severe & $\begin{array}{c}\text { IVS7-1G>A, p.Ala443Val, } \\
\text { p.Arg769Trp }\end{array}$ \\
\hline E160115 & $2016 / 8 / 27$ & 2,900 & $39+3$ & Female & Normal & 168 & $43 d$ & 25.89 & 6.95 & Moderate & p.Arg279Trp \\
\hline E160129 & 2016/8/30 & 4,100 & $37+4$ & Female & Goiter & 275 & $57 d$ & $>100$ & 1.42 & Severe & p.Glu337Lys \\
\hline E170009 & $2016 / 11 / 27$ & 3,900 & 40 & Female & Normal & 74 & $43 d$ & $>100$ & 4.76 & Severe & p.Asn592Ser, p.Asn798Lys \\
\hline E170026 & 2007/6/4 & NA & NA & Female & Normal & 10 & $20 d$ & 15.8 & 7.40 & Moderate & p.Ser853Leu \\
\hline E170076 & 2016/3/23 & NA & NA & Male & Normal & 15 & $22 d$ & 23 & 6.80 & Moderate & p.Glu673Lys \\
\hline
\end{tabular}

NA, not available; p.Glu757*, "was used to describe a stop codon. 
TABLE 2 | Detailed information of detected TPO variants.

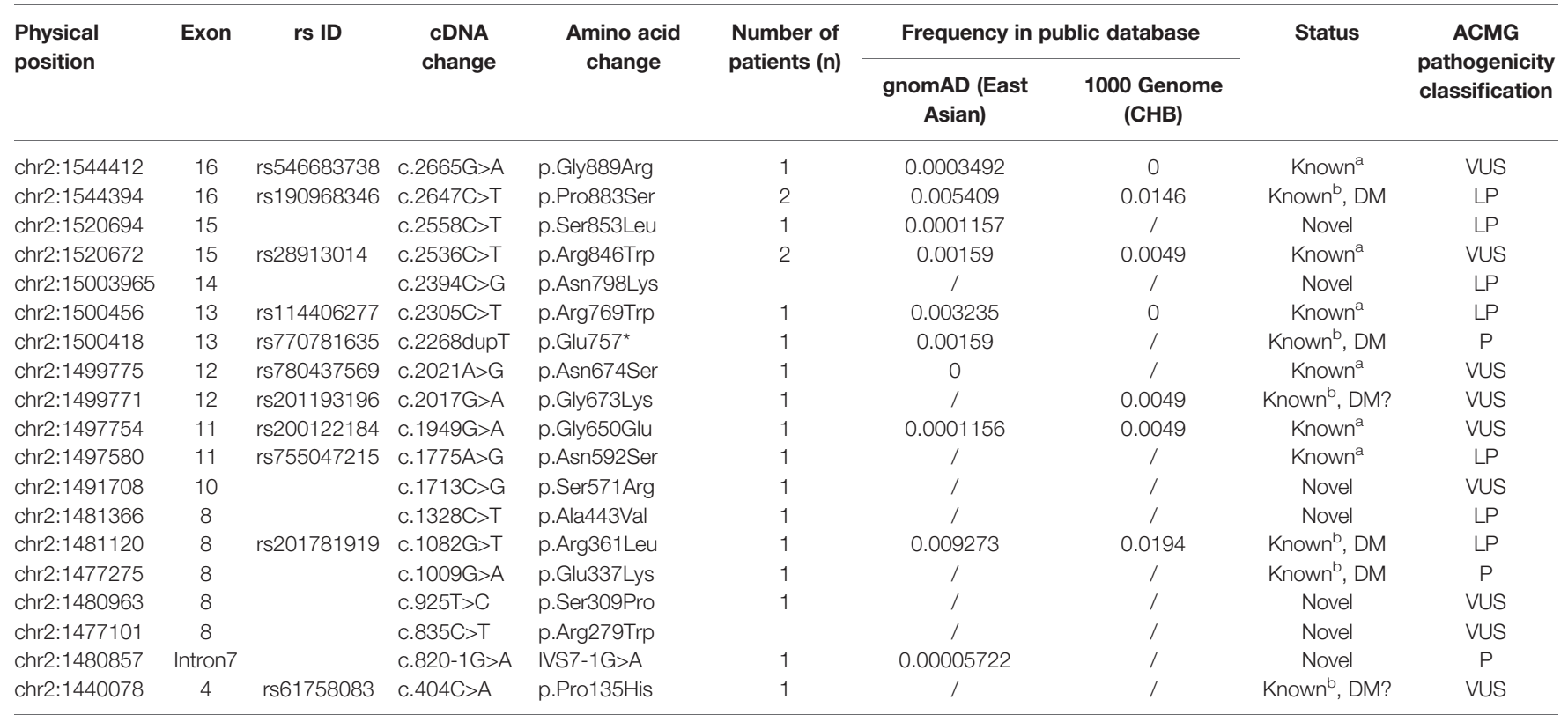

p.G/u757*, "was used to describe a stop codon.

${ }^{a}$ Variants were reported in public population databases, such as dbSNP, ExAC, or 1000 Genomes Project but without phenotypic data and pathological assessment.

${ }^{b}$ variants were reported in the published literature as well as HGMD (professional version 2021.2); DM, disease-causing mutation; DM?, a possible disease-causing mutation;

$P$, pathogenic; LP, likely pathogenic; VUS, variants of uncertain significance.
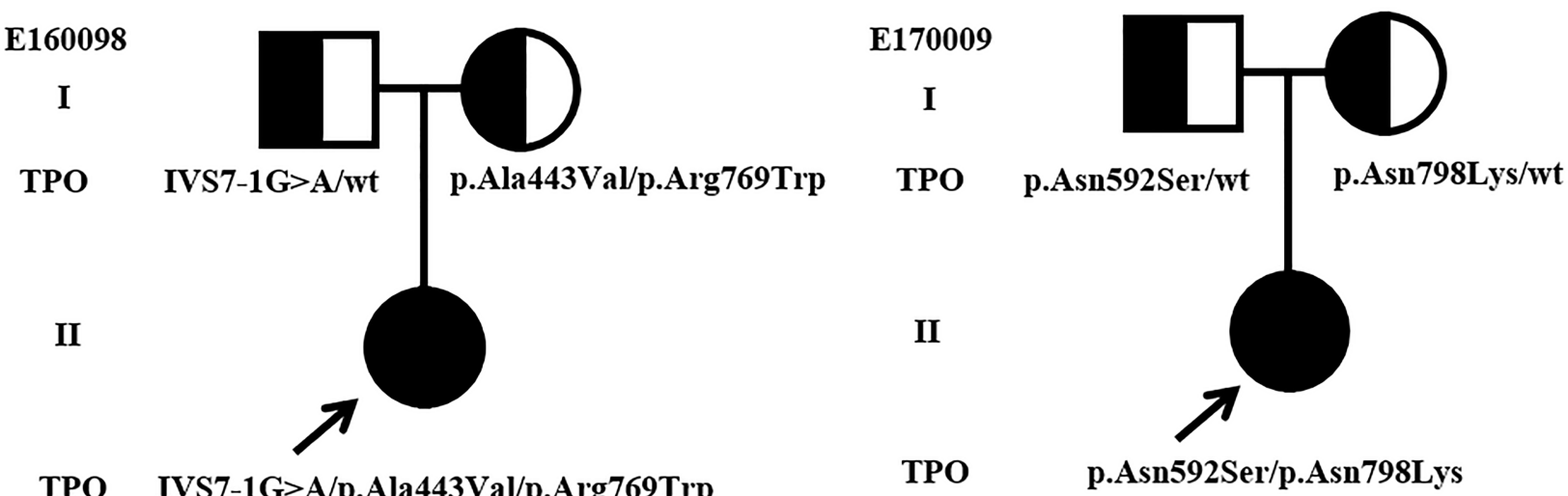

TPO p.Asn592Ser/p.Asn798Lys

FIGURE 1 | Genotypes and pedigrees of two CH patients. Arrow, Proband; Roman numbers, generations; squares, males; circles, females; half-filled symbols, unaffected heterozygote individuals.

Supplementary Figure 1). The comparison between predicted tertiary structures of wt and mutant proteins was performed. p.Asn798Lys, a neutral Asn replaced by the positively charged Lys, formed new interactions between the mutated residue Lys and surrounding residues, including covalent bond with $797 \mathrm{Val}$, hydrogen bonds with 797Val, 820Gly, and 821Phe, and salt bridge with Asp796. p.Arg769Trp, a positively charged Arg mutated to the neutral aromatic residue Trp, made the noncovalent interaction between Arg769 and Tyr772 disappear, resulting in new interactions between the mutated residue
Trp769 and surrounding residues Ala856 and Ala855. A strong interaction via the hydrogen bond network was observed between Asn592 and five surrounding residues (i.e., Glu596, Asp630, Arg595, Asp630, and Leu603). However, the replacement of Ser592 broke the hydrogen bond interactions with Arg595, Asp630, and Leu603. For p.Ala443Val, noncovalent interactions were observed between Ala443 and Val440, Gln446, Ile447, Phe678, and Leu686. When Ala443 was replaced by $\mathrm{Val}$, the hydrogen bond interactions with Gln446 and Ile447 were disappeared. 


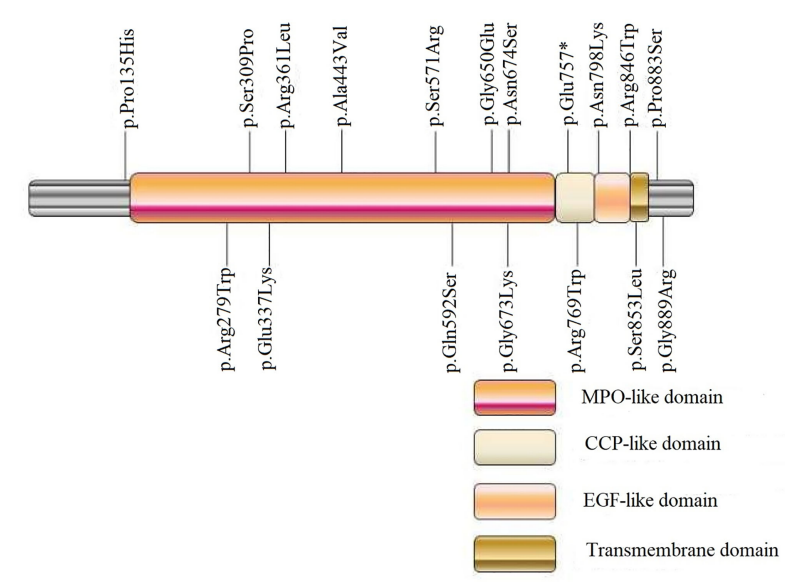

FIGURE 2 | Schematic diagram of detected exonic variants in TPO protein domains. MPO, myeloperoxidase; CCP, complement control protein; EGF, epidermal growth factor. p.Glu757*, * was used to describe a stop codon.

\section{In Vitro Functional Characterization of Mutant Proteins}

In vitro function studies were performed on 4 variants (i.e., p.Asn798Lys, p.Arg769Trp, p.Asn592Ser, and p.Ala443Val) carried by two compound heterozygotes (E160098 and E170009) and 1 documented pathogenic variant p.Glu337Lys. Immunoblotting experiment showed that the relative expression level of these five mutant proteins were comparable with that of the wt TPO protein ( $\mathrm{p}>0.05$, Figure 4).

Guaiacol was used as substrate, and the specific enzymatic activities of wt and mutant TPO proteins were determined. The specific activity of these five mutants were reduced to about $8-32 \%$ of that of wt protein, and the activities of p.Glu337Lys $(\mathrm{p}<0.05)$ and p.Ala443Val $(\mathrm{p}<0.05)$ were significantly decreased compared with that of the wt protein (Figure 5).

\section{DISCUSSION}

In this study, we screened and identified TPO gene mutations in 219 patients with $\mathrm{CH}$ from northwest China, which had a high incidence of $\mathrm{CH}$. The mutation spectrum of the TPO gene was analyzed, and the relationship between the TPO genotype and phenotype in patients with $\mathrm{CH}$ were explored to elucidate the pathogenic mechanism of TPO mutations in $\mathrm{CH}$ occurrence and provide a strong basis for the diagnosis, prognosis, and treatment of patients with $\mathrm{CH}$.

First, the mutation spectrum of the TPO gene in patients with $\mathrm{CH}$ was analyzed. Nineteen rare variants were identified in 17 patients with a detection rate of $7.8 \%$. This value was higher than the $1 \%$ TPO mutation rate reported in Guangxi located in the south of China (20) and lower than the rate of $10 \%$ in Shanghai located in the east of China (21) (Supplementary Table 3). Fifteen patients carried one heterozygous mutation, and two patients carried multiple TPO mutations. Based on the autosomal recessive pattern of the TPO mutation, data suggested that the TPO mutation might not be the major genetic factor causing $\mathrm{CH}$ in the Chinese population. In addition, c.2268dupT (p.E757fs or p.E757*), a non-sense mutation in the exon 13 of the TPO gene, is reported as a hotspot mutation in Taiwanese and Malaysian-Chinese patients and the most common genetic cause of dyshormonogenetic $\mathrm{CH}$ with evidence of cofounder effect (10, 30, 32). However, c.2268dupT was only found heterozygous in one patient of the present study, and all other identified variants demonstrated low frequency. These data validated an evident regional and ethnic difference in the role of TPO mutation in the pathogenesis of $\mathrm{CH}$.

Second, the relationship between the TPO genotype and phenotype of patients with $\mathrm{CH}$ in the studied cohort was studied. Previous studies showed that patients with $\mathrm{CH}$ and TPO mutations often presented with permanent $\mathrm{CH}$ and goiter $(9,11,15)$. In our study, the two patients carrying multiple TPO mutations had severe $\mathrm{CH}$ and FT4 levels below $5 \mathrm{pmol} / \mathrm{L}$, indicating that TPO biallelic mutations often led to severe $\mathrm{CH}$ phenotype. However, this study also confirmed that the TPO genotype was not directly related to the patient phenotype. The phenotype of patients carrying a single heterozygous variant remarkably varied, demonstrating light, moderate, and severe $\mathrm{CH}$. Even with the same mutation, the patient's phenotype was not identical. The thyroid morphology of patients was also varied. Some had goiter, some had normal or no thyroid, indicating that other undetected gene mutations or other factors participated in the final embodiment of the phenotype.

Third, the effects of identified variants on the TPO protein structure and function were investigated. Consistent with those in previous studies $(9,15,16,20,33,34)$, the majority of detected variants were located in the MPO-like domain of the TPO protein, which hamper normal biological functions. In patients with TPO mutations, two cases harbored multiple mutations, i.e., compounds for (1) IVS7-1G>A, p.Ala443Val, and p.Arg769Trp and (2) p.Asn592Ser and p.Asn798Lys. The variants they carried were of either paternal or maternal origin, and none came from one single parent. IVS7-1G>A is a splicing variant affecting the splicing of precursor mRNA, which may lead to the production of inactive protein. The functions of the four missense mutations (p.Ala443Val, p.Arg769Trp, p.Asn592Ser, and p.Asn798Lys) they carried have not been studied before. Thus, in silico methods and in vitro experiments were performed on these four variants and one previously documented pathogenic variant p.Glu337Lys. Experimental data showed that the protein expression levels of these five mutants were comparable with that of the wt TPO protein, but the enzymatic activities of these mutants were remarkably reduced. p.Asn798Lys, p.Asn592Ser, p.Ala443Val, and p.Glu337Lys only retained less than $20 \%$ activity toward guaiacol oxidation. Together with another TPO mutation, p.Glu337Lys was previously reported in a patient with a PIOD and goiter and showed residual activity towards guaiacol oxidation (11). In the present study, p.Glu337Lys was found in a patient with goiter, 

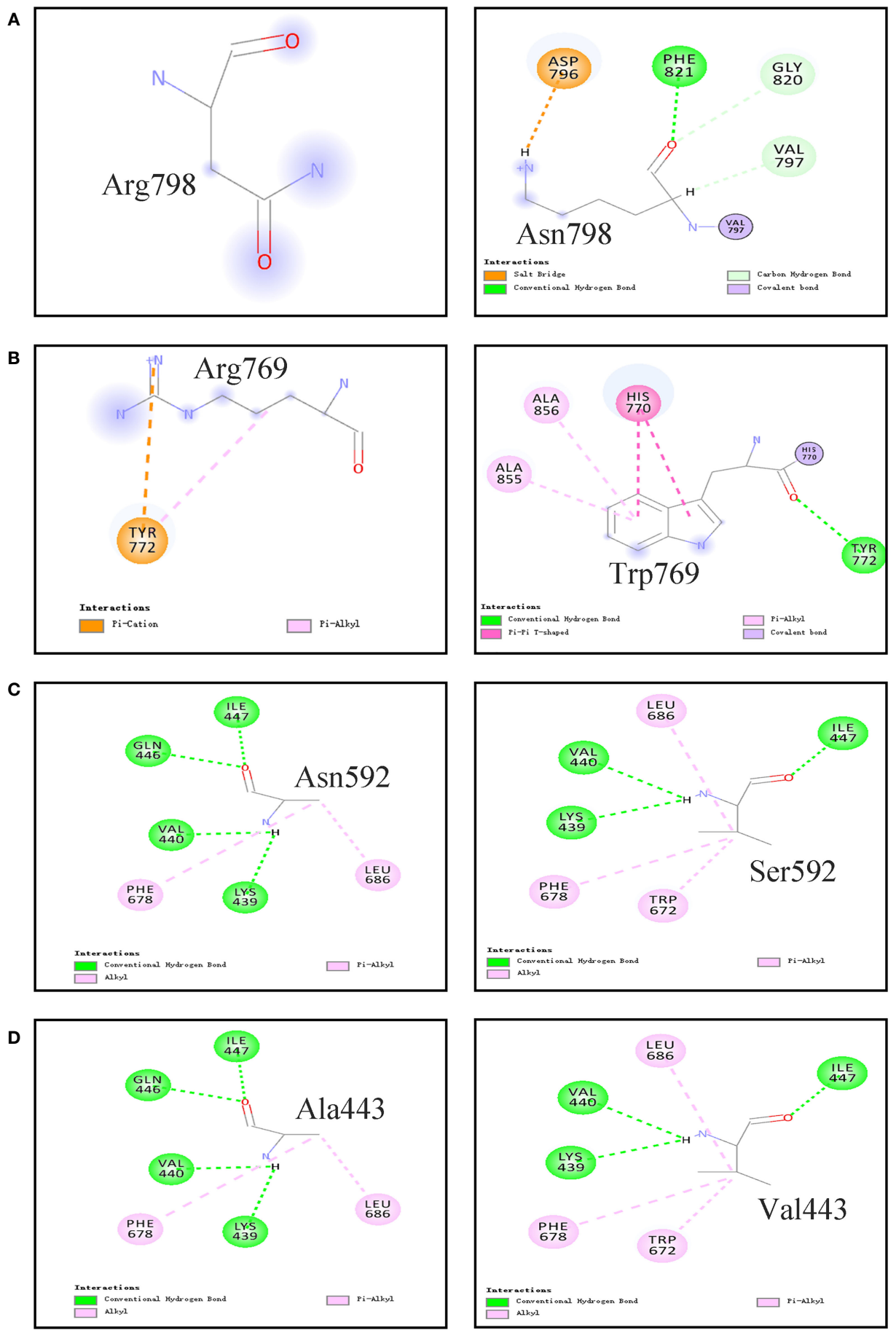

FIGURE 3 | Non-covalent Interactions between the studied residues with surrounding residues in the TPO protein which are generated by computer. (A) p.Asn798Arg; (B) p.Arg769Trp; (C) p.Asn592Ser; (D) p.Ala443Val.

and the experimental data reconfirmed its causal role. Ala443 and Asn592 were all located in the MPO-like domain of TPO protein and highly conserved in the TPO family and in other members of the peroxidase superfamily (Supplementary Figure 2). In silico programs predicted that p.Ala443Val and
p.Asn592Ser might have a deleterious effect on the TPO protein, and the molecular modeling analysis showed that the amino acid substitutions impaired multiple hydrogen-bonding interactions in the protein, which might destabilize the structure of protein. The mutant p.Arg769Trp, located in the CCP-like domain, a 


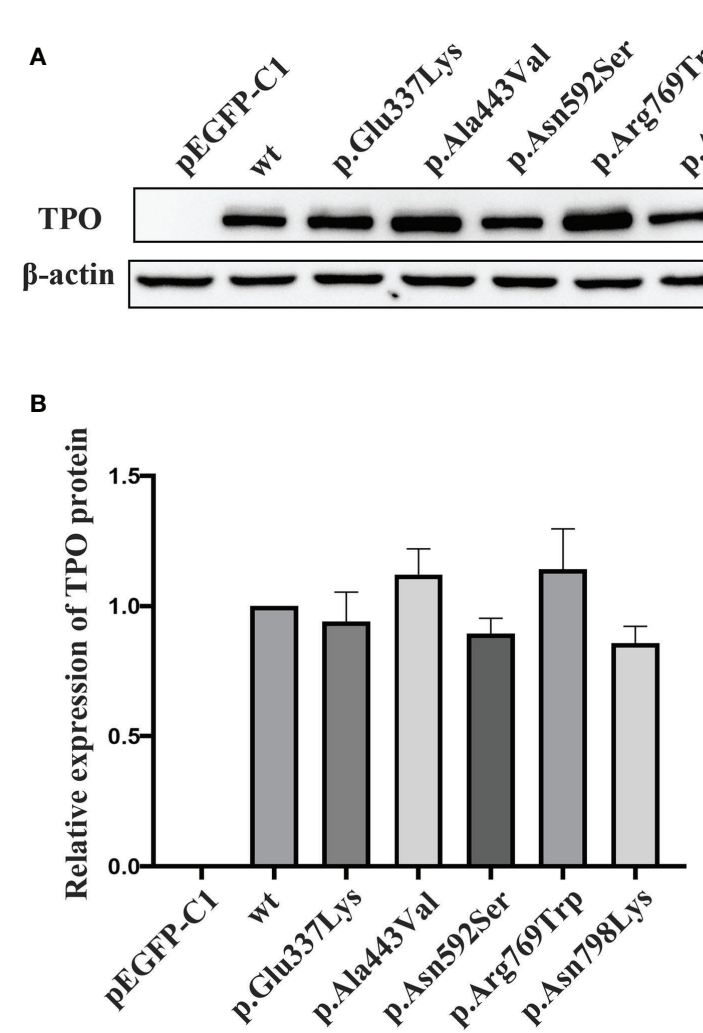

FIGURE 4 | Protein expression level of wild-type and mutant TPOs. (A) Western blotting results of wild-type and mutant TPO proteins. (B) Relative expression level of tested proteins in relation to wild-type TPO protein. wt, wild-type.

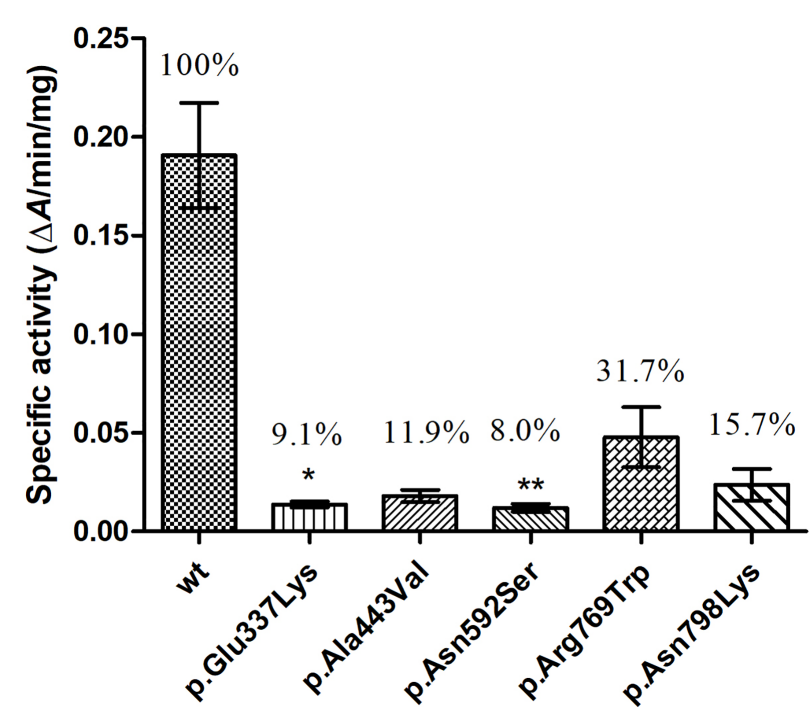

FIGURE 5 | Enzymatic activity of wild-type (wt) and mutant TPO proteins. ${ }^{*} P<0.05$; ${ }^{* *} P<0.01$. $P$ value refers to Dunnett's test, all compared with wild-type TPO protein. positively charged arginine is substituted by the neutral Trp, and the mutant p.Asn798Lys, located in the EGF-like domain, the neutral Asn is replaced by the positively charged Lys. The mutant forms of p.Arg769Trp and p.Asn798Lys all formed multiple new interactions with surrounding residues, which might change the protein conformation and catalytic activity. These findings suggested that these five missense variants might affect the protein function through the alteration of protein structure or conformation but not the protein expression. Based on these above data, we can preclude that compound heterozygous mutations identified in TPO were highly likely to be causal for $\mathrm{CH}$.

Several limitations should be addressed in this study. First, some patients in this study changed hospitals during treatment. Thus, complete clinical information was impossible to collect, thereby affecting the in-depth analysis of the TPO genotype-phenotype relationship. Second, only a minority of $\mathrm{CH}$ cases are genetic diseases caused by single genetic defects, and the majority of $\mathrm{CH}$ may be caused by multiple genetic and/or environmental factors $(2,35)$. Samples should be screened for other genes in subsequent studies to gain an improved understanding of the genetic etiology of $\mathrm{CH}$. Finally, biological function investigations were not performed on all detected variants, and we just paid attention to the variants validated through the family pedigree analysis. In the future, further studies with large sample size and pedigree samples are needed to verify the conclusions obtained in this study and gain a thorough understanding on the molecular mechanism of TPO mutation involved in the $\mathrm{CH}$ pathogenesis.

In summary, this study conducted a comprehensive screening and identification of TPO mutations in 219 patients with $\mathrm{CH}$ in northwest China and performed preliminary biological functional studies to reveal the pathogenic mechanism of some important variants. We found TPO mutations occurred at a low frequency in Chinese patients with $\mathrm{CH}$ and might not be the main genetic factor causing $\mathrm{CH}$. Functional studies of the five TPO mutations suggested that these amino acid substitution mutations might affect the protein function by changing the protein structure of the protein, which might result in impaired thyroid hormone synthesis.

\section{DATA AVAILABILITY STATEMENT}

The datasets presented in this study can be found in online repositories. The names of the repository/repositories and accession number(s) can be found in the article/Supplementary Material.

\section{ETHICS STATEMENT}

The studies involving human participants were reviewed and approved by Urumqi Maternal and Child Health Care Hospital. Written informed consent to participate in this study was provided by the participants' legal guardian/next of kin. 


\section{AUTHOR CONTRIBUTIONS}

HW and GD conceptualized and designed the study and drafted the initial manuscript. XC collected the sample and analyzed the basic clinical data. WW and YS performed the experiments. HS and HW analyzed the data and interpreted the results. All authors contributed to the article and approved the submitted version of the manuscript and agreed to be accountable for all aspects of the work.

\section{FUNDING}

This work was supported by the Natural Science Foundation of Xinjiang Uygur Autonomous Region (2019D01A16).

\section{ACKNOWLEDGMENTS}

We would like to thank all the patients and staffs (e.g. Yanwei LI, Min Han and Jiying Ma) involved in the experiment.

\section{REFERENCES}

1. Rastogi MV, LaFranchi SH. Congenital Hypothyroidism. Orphanet J Rare Dis (2010) 5:17. doi: 10.1186/1750-1172-5-17

2. Wassner AJ, Brown RS. Congenital Hypothyroidism: Recent Advances. Curr Opin Endocrinol Diabetes Obes (2015) 22(5):407-12. doi: 10.1097/MED. 0000000000000181

3. Nettore IC, Cacace V, De Fusco C, Colao A, Macchia PE. The Molecular Causes of Thyroid Dysgenesis: A Systematic Review. J Endocrinol Invest (2013) 36(8):654-64. doi: 10.3275/8973

4. Grasberger H, Refetoff S. Genetic Causes of Congenital Hypothyroidism Due to Dyshormonogenesis. Curr Opin Pediatr (2011) 23(4):421-8. doi: 10.1097/ MOP.0b013e32834726a4

5. van Trotsenburg P, Stoupa A, Leger J, Rohrer T, Peters C, Fugazzola L, et al. Congenital Hypothyroidism: A 2020-2021 Consensus Guidelines Update-An ENDO-European Reference Network Initiative Endorsed by the European Society for Pediatric Endocrinology and the European Society for Endocrinology. Thyroid (2021) 31(3):387-419. doi: 10.1089/thy.2020.0333

6. Stoupa A, Kariyawasam D, Muzza M, de Filippis T, Fugazzola L, Polak M, et al. New Genetics in Congenital Hypothyroidism. Endocrine (2021) 71 (3):696-705. doi: 10.1007/s12020-021-02646-9

7. Wassner AJ. Unraveling the Genetics of Congenital Hypothyroidism: Challenges and Opportunities. J Clin Endocrinol Metab (2020) 105(9): dgaa454. doi: 10.1210/clinem/dgaa454

8. Baker JR, Arscott P, Johnson J. An Analysis of the Structure and Antigenicity of Different Forms of Human Thyroid Peroxidase. Thyroid (1994) 4(2):173-8. doi: $10.1089 /$ thy.1994.4.173

9. Rivolta CM, Esperante SA, Gruneiro-Papendieck L, Chiesa A, Moya CM, Domene S, et al. Five Novel Inactivating Mutations in the Thyroid Peroxidase Gene Responsible for Congenital Goiter and Iodide Organification Defect. Hum Mutat (2003) 22(3):259. doi: 10.1002/humu.9175

10. Niu DM, Hwang B, Chu YK, Liao CJ, Wang PL, Lin CY. High Prevalence of a Novel Mutation (2268 Inst) of the Thyroid Peroxidase Gene in Taiwanese Patients With Total Iodide Organification Defect, and Evidence for a Founder Effect. J Clin Endocrinol Metab (2002) 87(9):4208-12. doi: 10.1210/jc.2002020153

11. Zhang J, Han R, Shen L, Xie J, Xiao Y, Jiang L, et al. Mild TPO Deficiency Characterized by Progressive Goiter and Normal Serum TSH Level. Endocrine (2020) 68(3):599-606. doi: 10.1007/s12020-020-02224-5

12. Bakker B, Bikker H, Vulsma T, de Randamie JS, Wiedijk BM, De Vijlder JJ. Two Decades of Screening for Congenital Hypothyroidism in the

\section{SUPPLEMENTARY MATERIAL}

The Supplementary Material for this article can be found online at: https://www.frontiersin.org/articles/10.3389/fendo.2021. 774941/full\#supplementary-material

Supplementary Figure 1 | Computer generated models of the wild type and mutant TPO. Ribbon (A, C, E, G) and Stick (B, D, F, H) presentation of wild-type and mutant TPO proteins [(A, B) p.Asn798Arg; (C, D) p.Arg769Trp; (E, F) p.Asn592Ser; (G, H) p.Ala443Val]. These four selected residues as well as those that have an interaction with them are shown as sticks. Non-covalent interactions are shown as dashed lines.

Supplementary Figure 2 | Comparisons of the amino acid sequences neighboring the mutations among different species, and different members of peroxidase superfamilies.

Supplementary Table 1 | Classification and evidence of detected TPO variants.

Supplementary Table 2 | In silico prediction of the function of detected TPO variants.

Supplementary Table 3 | Comparison of studies that conducted TPO screening in patients with $\mathrm{CH}$.

Netherlands: TPO Gene Mutations in Total Iodide Organification Defects (an Update). J Clin Endocrinol Metab (2000) 85(10):3708-12. doi: 10.1210/ jcem.85.10.6878

13. Bikker H, Baas F, De Vijlder JJ. Molecular Analysis of Mutated Thyroid Peroxidase Detected in Patients With Total Iodide Organification Defects. J Clin Endocrinol Metab (1997) 82(2):649-53. doi: 10.1210/jcem.82.2.3729

14. Kotani T, Umeki K, Kawano J, Suganuma T, Hishinuma A, Ieiri T, et al. Partial Iodide Organification Defect Caused by a Novel Mutation of the Thyroid Peroxidase Gene in Three Siblings. Clin Endocrinol (Oxf) (2003) 59 (2):198-206. doi: 10.1046/j.1365-2265.2003.01823.x

15. Avbelj M, Tahirovic H, Debeljak M, Kusekova M, Toromanovic A, Krzisnik C, et al. High Prevalence of Thyroid Peroxidase Gene Mutations in Patients With Thyroid Dyshormonogenesis. Eur J Endocrinol (2007) 156(5):511-9. doi: 10.1530/EJE-07-0037

16. Cangul H, Aycan Z, Olivera-Nappa A, Saglam H, Schoenmakers NA, Boelaert $\mathrm{K}$, et al. Thyroid Dyshormonogenesis Is Mainly Caused by TPO Mutations in Consanguineous Community. Clin Endocrinol (Oxf) (2013) 79(2):275-81. doi: $10.1111 /$ cen.12127

17. Lof C, Patyra K, Kuulasmaa T, Vangipurapu J, Undeutsch H, Jaeschke H, et al. Detection of Novel Gene Variants Associated With Congenital Hypothyroidism in a Finnish Patient Cohort. Thyroid (2016) 26(9):121524. doi: $10.1089 /$ thy.2016.0016

18. Narumi S, Muroya K, Asakura Y, Aachi M, Hasegawa T. Molecular Basis of Thyroid Dyshormonogenesis: Genetic Screening in Population-Based Japanese Patients. J Clin Endocrinol Metab (2011) 96(11):E1838-42. doi: 10.1210/jc.2011-1573

19. Chen X, Kong X, Zhu J, Zhang T, Li Y, Ding G, et al. Mutational Spectrum Analysis of Seven Genes Associated With Thyroid Dyshormonogenesis. Int J Endocrinol (2018) 2018:8986475. doi: 10.1155/2018/8986475

20. Fu C, Xie B, Zhang S, Wang J, Luo S, Zheng H, et al. Mutation Screening of the TPO Gene in a Cohort of 192 Chinese Patients With Congenital Hypothyroidism. BMJ Open (2016) 6(5):e010719. doi: 10.1136/bmjopen2015-010719

21. Zhang RJ, Sun F, Chen F, Fang Y, Yan CY, Zhang CR, et al. The TPO Mutation Screening and Genotype-Phenotype Analysis in 230 Chinese Patients With Congenital Hypothyroidism. Mol Cell Endocrinol (2020) 506:110761. doi: 10.1016/j.mce.2020.110761

22. Lee ST, Lee DH, Kim JY, Kwon MJ, Kim JW, Hong YH, et al. Molecular Screening of the TSH Receptor (TSHR) and Thyroid Peroxidase (TPO) Genes in Korean Patients With Nonsyndromic Congenital Hypothyroidism. Clin Endocrinol (Oxf) (2011) 75(5):715-21. doi: 10.1111/j.1365-2265.2011.04156.x 
23. Wang F, Zang Y, Li M, Liu W, Wang Y, Yu X, et al. DUOX2 and DUOXA2 Variants Confer Susceptibility to Thyroid Dysgenesis and Gland-In-Situ With Congenital Hypothyroidism. Front Endocrinol (Lausanne) (2020) 11:237. doi: 10.3389/fendo.2020.00237

24. Deng K, He C, Zhu J, Liang J, Li X, Xie X, et al. Incidence of Congenital Hypothyroidism in China: Data From the National Newborn Screening Program, 2013-2015. J Pediatr Endocrinol Metab (2018) 31(6):601-8. doi: 10.1515/jpem-2017-0361

25. Yu B, Long W, Yang Y, Wang Y, Jiang L, Cai Z, et al. Newborn Screening and Molecular Profile of Congenital Hypothyroidism in a Chinese Population. Front Genet (2018) 9:509. doi: 10.3389/fgene.2018.00509

26. Xue S, Ma G, Liu N, Li Z. Analysis on the Results of 45499 Neonates Screening for Congenital Hypothyroidism in Xinjiang. Chin J Birth Health Hered (2011) 19(7):82-3. doi: 10.13404/j.cnki.cjbhh.2011.07.053

27. Wang H, Kong X, Pei Y, Cui X, Zhu Y, He Z, et al. Mutation Spectrum Analysis of 29 Causative Genes in 43 Chinese Patients With Congenital Hypothyroidism. Mol Med Rep (2020) 22(1):297-309. doi: 10.3892/mmr.2020.11078

28. Richards S, Aziz N, Bale S, Bick D, Das S, Gastier-Foster J, et al. Standards and Guidelines for the Interpretation of Sequence Variants: A Joint Consensus Recommendation of the American College of Medical Genetics and Genomics and the Association for Molecular Pathology. Genet Med (2015) 17(5):405-24. doi: 10.1038/gim.2015.30

29. Belforte FS, Targovnik AM, Gonzalez-Lebrero RM, Osorio Larroche C, Citterio CE, Gonzalez-Sarmiento R, et al. Kinetic Characterization of Human Thyroperoxidase. Normal and Pathological Enzyme Expression in Baculovirus System: A Molecular Model of Functional Expression. Mol Cell Endocrinol (2015) 404:9-15. doi: 10.1016/j.mce.2014.12.026

30. Lee CC, Harun F, Jalaludin MY, Heh CH, Othman R, Junit SM. Prevalence of C.2268dup and Detection of Two Novel Alterations, C.670_672del and C.1186C $>\mathrm{T}$, in the TPO Gene in a Cohort of Malaysian-Chinese With Thyroid Dyshormonogenesis. BMJ Open (2015) 5(1):e006121. doi: 10.1136/ bmjopen-2014-006121

31. Leger J, Olivieri A, Donaldson M, Torresani T, Krude H, van Vliet G, et al. European Society for Paediatric Endocrinology Consensus Guidelines on
Screening, Diagnosis, and Management of Congenital Hypothyroidism. Horm Res Paediatr (2014) 81(2):80-103. doi: 10.1159/000358198

32. Wu JY, Shu SG, Yang CF, Lee CC, Tsai FJ. Mutation Analysis of Thyroid Peroxidase Gene in Chinese Patients With Total Iodide Organification Defect: Identification of Five Novel Mutations. J Endocrinol (2002) 172(3):627-35. doi: $10.1677 /$ joe.0.1720627

33. Rodrigues C, Jorge P, Soares JP, Santos I, Salomao R, Madeira M, et al. Mutation Screening of the Thyroid Peroxidase Gene in a Cohort of 55 Portuguese Patients With Congenital Hypothyroidism. Eur J Endocrinol (2005) 152(2):193-8. doi: 10.1530/eje.1.01826

34. Targovnik HM, Citterio CE, Rivolta CM. Iodide Handling Disorders (NIS, TPO, TG, IYD). Best Pract Res Clin Endocrinol Metab (2017) 31(2):195-212. doi: 10.1016/j.beem.2017.03.006

35. Kostopoulou E, Miliordos K, Spiliotis B. Genetics of Primary Congenital Hypothyroidism-a Review. Hormones (Athens) (2021) 20(2):225-36. doi: $10.1007 /$ s42000-020-00267-x

Conflict of Interest: The authors declare that the research was conducted in the absence of any commercial or financial relationships that could be construed as a potential conflict of interest.

Publisher's Note: All claims expressed in this article are solely those of the authors and do not necessarily represent those of their affiliated organizations, or those of the publisher, the editors and the reviewers. Any product that may be evaluated in this article, or claim that may be made by its manufacturer, is not guaranteed or endorsed by the publisher.

Copyright (c) 2021 Wang, Wang, Chen, Shi, Shi and Ding. This is an open-access article distributed under the terms of the Creative Commons Attribution License (CC BY). The use, distribution or reproduction in other forums is permitted, provided the original author(s) and the copyright owner(s) are credited and that the original publication in this journal is cited, in accordance with accepted academic practice. No use, distribution or reproduction is permitted which does not comply with these terms. 